\title{
Análise do banco de sementes e da fertilidade do solo como ferramentas para recuperação de áreas perturbadas
}

\author{
Janaína Oliveira da Silva ${ }^{1 *}$ \\ Evandro Binotto Fagan ${ }^{1}$ \\ Walquíria Fernanda Teixeira ${ }^{2}$ \\ Marília Caixeta Sousa ${ }^{1}$ \\ Juliana Rosa Silva ${ }^{3}$ \\ ${ }^{1}$ Centro Universitário de Patos de Minas, Patos de Minas - MG, Brasil \\ ${ }^{2}$ Universidade Federal de Uberlândia, Uberlândia, MG, Brasil \\ ${ }^{3}$ Universidade Federal de Lavras, Lavras - MG, Brasil \\ *Autor para correspondência \\ janainapreta@hotmail.com \\ Rua Formiga, 33, CEP 38701-024, Patos de Minas - MG, Brasil
}

Submetido em 11/06/2011

Aceito para publicação em 04/10/2011

\section{Resumo}

A capacidade de perpetuação das espécies de uma floresta depende da viabilidade do banco de sementes e fertilidade do solo. Este trabalho teve como objetivo analisar a estrutura do banco de sementes e as condições do solo como ferramentas para recuperação de uma área perturbada do Parque Municipal do Mocambo de Patos de Minas, MG. O local foi dividido em três áreas sendo que, em cada área, foram selecionadas aleatoriamente nove parcelas de $10 \times 30 \mathrm{~m}\left(300 \mathrm{~m}^{2}\right)$ e em cada uma dessas parcelas foram coletadas amostras de solo em três pontos e três profundidades diferentes. A metade destas amostras foi mantida sobre a irradiação de luz e o restante no escuro. Uma amostra do solo de cada área foi coletada para se verificar a fertilidade do solo. Observou-se dominância de espécies herbáceas pioneiras nas emergências. O maior índice de emergência foi encontrado na profundidade de 0 a $10 \mathrm{~cm}$. O solo mostrou-se com deficiência em potássio e fósforo, sendo que nos demais nutrientes analisados os valores encontravam-se muito bons. Conclui-se que, em longo prazo, essa área consiga se regenerar naturalmente, e é possível também acelerar esse processo com a introdução de espécies nativas e de dispersores naturais.

Palavras-chave: Análise do Solo, Emergência, Parque Mocambo, Regeneração

\section{Abstract}

Analysis of the seed bank and soil fertility as tools for recovery of disturbed areas. The perpetuation of plant species in forests depends on the viability of the seed bank and soil fertility. The goal of this study was to verify the species present in the seed bank, and the soil conditions that are available for use during recovery, of a disturbed area in Parque Municipal do Mocambo de Patos de Minas, MG. The site was divided into three areas and each area was further divided into nine random parcels that were $10 \times 30 \mathrm{~m}\left(300 \mathrm{~m}^{2}\right)$. Within each parcel, soil samples were collected in three places and three depths. Half of the samples were kept in the light and the 
other half in the dark. A soil sample from each area was collected to determine soil fertility. Herbaceous pioneer species emerged the most and the soil depth between $0-10 \mathrm{~cm}$ had the highest emergency index. The soil tested was deficient in potassium and phosphorus; however, the values for other nutrients were good. This work found that the area studied can regenerate naturally, but it would be possible to speed up that process by introducing native plant species and native dispersers into area.

Key words: Emergence, Parque Mocambo, Regeneration, Soil analysis

\section{Introdução}

O Cerrado é o segundo maior bioma brasileiro, com uma extensão de 1,8 milhões de $\mathrm{km}^{2}$, e também o maior do estado de Minas Gerais, constituído por mosaicos de formações vegetais que variam desde campos abertos até formações densas de florestas (AGUIAR; CAMARGO, 2004). Entretanto, estudos recentes comprovam que apenas $20 \%$ da área total são consideradas originais ou pouco perturbadas. Isto se deve a vários fatores, como a urbanização, que além de modificar a paisagem, também provoca aumento da erosão do solo, alterando suas características físicas e químicas, alterando os processos naturais dos ecossistemas (AGUIAR; CAMARGO, 2004).

A restauração de áreas perturbadas inicia-se através da avaliação do solo, da estrutura vegetativa e de um levantamento florístico. Todas essas etapas possibilitam a identificação das características do ambiente perturbado e dos mecanismos que serão utilizados para a sua revitalização. O estabelecimento das funções ecológicas destes ecossistemas ocorre principalmente através da deposição da serrapilheira (folhas e galhos em decomposição, microrganismos, restos animais, sementes), da chuva de sementes, do banco de sementes e da regeneração natural (PINTO JÚNIOR, 2008).

A utilização de uma nova dinâmica de sucessão ecológica é fundamental para o estabelecimento de novas espécies. Essa dinâmica pode ser compreendida pela modificação do ambiente físico pela comunidade e controlada pela mesma, onde também o ambiente orienta a extensão do desenvolvimento, buscando alcançar o clímax, que é o ecossistema estabilizado (ODUM, 1988).

Há várias maneiras de adotar uma sucessão ecológica, e estas podem ser aceleradas por meio de estratégias baseadas em princípios nucleares de vegetação. A nucleação proporciona uma sucessão natural através das relações interespecíficas, com uma alta dinâmica das comunidades, gerando resultados em curto prazo (BRECHARA et al., 2007). Destacamse como técnicas nucleadoras a transposição de solo para restituição do banco de sementes e biota do solo, o plantio de mudas de espécies arbóreas em grupos, formando núcleos para eliminação de gramíneas exóticas invasoras e a formação de coberturas de solo através de semeadura direta de espécies herbáceas e arbustivas, preferencialmente nativas, entre outras (TOMAZI et al., 2010).

As áreas que sofreram algum tipo de exploração podem ser classificadas em degradadas ou perturbadas. Um ambiente perturbado é aquele que sofreu algum tipo de distúrbio, mas manteve os seus meios de regeneração (banco de sementes, banco de plântulas, chuva de sementes). Denomina-se como degradado aquele ambiente que após distúrbios teve eliminado, com a vegetação, os seus meios de regeneração biótica (REZENDE, 1998).

Em áreas degradadas ou perturbadas, o estudo do banco de sementes viabiliza a implantação de projetos e alternativas que auxiliem na recuperação dessas áreas, visando à estruturação e estabilidade de um ecossistema (NÓBREGA et al., 2009).

O banco de sementes é um sistema dinâmico, através da entrada de sementes através da chuva de sementes e dispersão, podendo ser transitório, com sementes que germinam dentro de um ano após o início da dispersão, ou persistente, com sementes que permanecem no solo por mais de um ano (CALDATO et al., 1996). O banco de sementes é definido como o conjunto de sementes viáveis presentes no solo ou subsolo que estão em dormência, indicando se há a possibilidade de utilização do banco 
de sementes do solo ou a introdução de novas espécies (VIEIRA; REIS, 2003).

No entanto, um dos grandes problemas encontrado em restaurações de áreas perturbadas é a proliferação de plantas oportunistas e herbáceas pioneiras, que impedem que o banco de sementes presente no solo possa se desenvolver, interferindo no desenvolvimento do ecossistema. Entretanto, as herbáceas pioneiras também são fundamentais no processo de sucessão, atuando no primeiro estágio de sucessão após uma perturbação (ARAÚJO et al., 2004).

O investimento em pesquisas sobre revitalização de áreas perturbadas aumentou a divulgação das questões ecológicas globais, ampliando a participação popular em relação à qualidade de vida humana e o meio ambiente. Portanto, vem aumentando as pressões junto aos governos para a constituição de políticas conservacionistas, que contribuam para aumento do número e extensão das áreas protegidas (VALLEJO, 2003).

Portanto, este trabalho teve como objetivo avaliar o banco de sementes em diferentes níveis de profundidade do solo na área perturbada do Parque Municipal do Mocambo, a fertilidade do solo como ferramentas para recuperação da mesma, e a capacidade de regeneração natural.

\section{Material e Métodos}

O experimento foi realizado em uma área perturbada do Parque Municipal do Mocambo, localizado na cidade de Patos de Minas - MG no período de abril de 2009 a agosto de 2009. O parque, criado em 1990, possui cerca de 7 ha em sua extensão e tem por finalidade promover atividades sócio-culturais e proteção de seus recursos naturais, uma vez que se localiza em área de preservação permanente (PATOS DE MINAS, 1990).

Para a demarcação das áreas de coleta, foi observada a existência de diferenças fisionômicas na vegetação, dividindo-a em três subáreas, $\mathrm{A}_{1}$ (topo), $\mathrm{A}_{2}$ (encosta) e $\mathrm{A}_{3}$ (baixada). Em cada subárea, foram demarcadas nove parcelas de $10 \mathrm{~m}$ de largura por $30 \mathrm{~m}$ de comprimento $\left(A_{1 n} ; A_{2 n} ; A_{3 n}\right)$.
O delineamento experimental utilizado foi o esquema $3 \times 3$, sendo três áreas de coleta e três profundidades de solo, $\mathrm{S}_{1}(0$ a $10 \mathrm{~cm}), \mathrm{S}_{2}(10$ a $20 \mathrm{~cm})$ e $\mathrm{S}_{3}(20$ a $30 \mathrm{~cm})$ com nove repetições. Em cada uma das parcelas foram abertas trincheiras de $40 \mathrm{~cm}$ de profundidade, com auxílio de enxada e enxadão, totalizando 162 amostras, onde 81 foram destinadas para germinação na presença de luz constante, e as outras 81 amostras foram mantidas no escuro. Foram coletadas nove amostras de solo de $500 \mathrm{~g}$ em cada área, para análise de fertilidade.

As amostras coletadas foram armazenadas em sacos plásticos identificados de acordo com a profundidade de coleta e em seguida levadas ao laboratório de Fisiologia Vegetal da Faculdade de Engenharias e Ciências Agrárias (FAECIA). Em cada amostra foram retirados $500 \mathrm{~g}$ de solo e colocados em bandejas plásticas de dimensões de $14 \times 18 \mathrm{~cm}$.

As bandejas plásticas foram identificadas de acordo com a profundidade de coleta do solo e área específica, possuindo em seu fundo uma camada de areia lavada de $3 \mathrm{~cm}$ para auxiliar a drenagem da água de irrigação no recipiente. E em seguida, foram colocadas em uma sala de germinação no Laboratório de Fisiologia Vegetal.

$\mathrm{Na}$ sala de germinação, metade das amostras foi mantida na presença de luz constante, com lâmpadas fluorescentes de $20 \mathrm{~W}$, a uma distância de $55 \mathrm{~cm}$ das bandejas, totalizando uma potência de $296 \mathrm{~W} \cdot \mathrm{m}^{-2}$. A outra metade foi mantida no escuro, foram cobertas por um plástico preto com espessura de 25 micra para impedir a incidência luminosa.

Diariamente, as bandejas foram umedecidas (50ml por dia de água), e observado o surgimento de germinação das sementes. Essa contagem das emergências é mais viável, pois permite que se obtenha a porção das sementes germinadas (GASPARINO et al., 2006). Foi considerado como critério de germinação o aparecimento das radículas e a propulsão da casca, sendo anotada e datada de acordo com o aparecimento. À medida que as características morfológicas foliares foram definidas, as espécies foram identificadas através do uso do catálogo descrito por Lorenzi (2000). 
As amostras de solo coletadas foram encaminhadas para a análise de sua fertilidade foram encaminhadas ao Laboratório de Solos da FAECIA.

Para a análise estatística dos dados, foi utilizado o teste de variância não paramétrico de Kruskal-Wallis através do software Assistat (SILVA, 1996).

\section{Resultados e Discussão}

A partir do segundo dia após a montagem do experimento foram observados o inicio da germinação de várias espécies nas três vegetações $\left(A_{1}, A_{2}\right.$ e $\left.A_{3}\right)$. Além disso, houve germinação nas três profundidades de solo coletados durante os 150 dias de observação. Entre as 193 plântulas germinadas sobre incidência de luz constante, 118 germinaram no solo coletado na profundidade de $0-10 \mathrm{~cm}\left(\mathrm{~S}_{1}\right)$ e, na profundidade de $10-20 \mathrm{~cm}\left(\mathrm{~S}_{2}\right)$, foram 52 emergências, e houve 23 na profundidade de $20-30 \mathrm{~cm}\left(\mathrm{~S}_{3}\right)$. Baider et al. (1999) também observaram maiores quantidades de sementes germinadas em solos superficiais.

No presente trabalho, todas as espécies germinadas foram pioneiras herbáceas pertencentes às famílias Asteraceae, Cyperaceae, Malvaceae, Commelinaceae, Rubiaceae e Euphorbiaceae, sendo que o maior número de indivíduos observados foram com a família Asteraceae (Figura 1). Estudos realizados por Baider et al. (1999) e Vinha (2008) sobre o banco de sementes em áreas perturbadas de Mata Atlântica também encontraram a presença dessas famílias, sendo estas importantes à colonização, pois facilitam o estabelecimento de outras, agindo como abrigo para os vetores de dispersão, melhorando as condições de fertilidade do solo e fornecendo habitats adequados à sucessão.

As espécies com maior índice de emergências foram a Ageratum conyzoides L., Conyza canadensis (L.) Cronquist., Acanthospermum hispidum DC., Brachiaria sp., Galinsoga parviflora Cav., Cyperus iria L. entre outras identificadas. A pequena quantidade de espécies secundárias encontradas no banco de sementes pode estar relacionada com a presença de dormência ou pela baixa presença de propágulos contidos neste levantamento florístico.
FIGURA 1: Distribuição do número de plântulas por famílias encontradas nas amostras de solo coletadas na área perturbada do Parque Municipal do Mocambo. UNIPAM, Patos de Minas, MG, 2009.

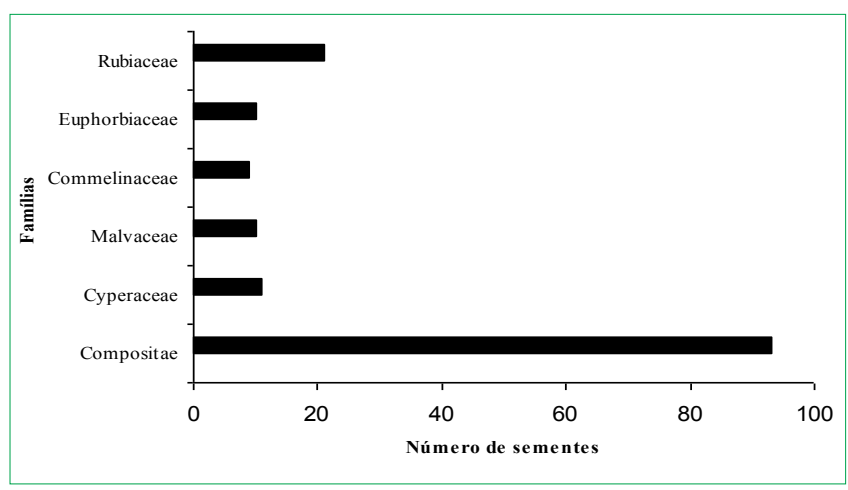

Analisando a quantidade de sementes germinadas, foi possível perceber que na profundidade $S_{1}$ ocorreu a maior taxa de emergência nas três sub-áreas avaliadas, com valores de 2,55 em $\mathrm{A}_{1}, 3,44$ na área 2 e 7,11 na área 3, seguidos pelas profundidades $\mathrm{S}_{2}$ e $\mathrm{S}_{3}$ (Tabela 1).

TABELA 1: Médias da taxa de germinação nas áreas $\mathrm{A}_{1}$, $A_{2}$ e $A_{3}$, correspondentes a área degradada do Parque do Mocambo em diferentes profundidades de solo. $\mathrm{S}_{1}$ correspondente ao solo coletado entre $0-10 \mathrm{~cm}, \mathrm{~S}_{2}$ entre $10-20 \mathrm{~cm}$ e $\mathrm{S}_{3}$ de $20-30 \mathrm{~cm}$.

\begin{tabular}{cccc}
\hline \multicolumn{4}{c}{ Áreas submetidas à luz } \\
\hline Profundidade & $\mathbf{A}_{1}$ & $\mathbf{A}_{2}$ & $\mathbf{A}_{3}$ \\
\hline $\mathbf{S}_{1}$ & $2,55 \mathrm{a}^{*}$ & $3,44 \mathrm{a}$ & $7,11 \mathrm{a}$ \\
$\mathbf{S}_{2}$ & $0,77 \mathrm{~b}$ & $2,22 \mathrm{~b}$ & $2,77 \mathrm{~b}$ \\
$\mathbf{S}_{3}$ & $0,77 \mathrm{~b}$ & $0,44 \mathrm{c}$ & $1,44 \mathrm{c}$ \\
\hline
\end{tabular}

* Médias seguidas pelas mesmas letras não diferem entre si pelo teste Kruskal-Wallis.

Analisando o banco de sementes nas diferentes profundidades, pode-se ainda inferir que a capacidade da área em se regenerar naturalmente está ligada basicamente à presença de espécies pioneiras herbáceas. É possível verificar que a profundidade de $0-10 \mathrm{~cm}$ é a que mais influencia efetivamente no banco de sementes, corroborando com os resultados observados por Miranda et al. (2007). Segundo Araújo et al. (2004), a expressiva quantidade de sementes de plantas pioneiras presentes no solo justifica-se pela produção contínua ou anual de propágulos dessas espécies. Isso justifica a presença 
de espécies pioneiras nas germinações analisadas, o que também foi observado por Nóbrega et al. (2009). Estas são aptas a suportar condições adversas, tolerar elevadas e baixas temperaturas, ambientes úmidos e secos e variações do suprimento de oxigênio, apresentando grande capacidade de produzir sementes (CHRISTOFFOLETE; CAETANO, 1998).

Segundo Almeida-Cortez (2004), áreas de vegetação próximas tendem a ter o mesmo tipo de espécies, pois as sementes de um local podem ser levadas para outro local próximo através de várias formas de dispersão como o vento, água, animais, ou por mecanismos próprios da planta.

Poucas sementes germinaram no escuro, ocorrendo apenas nas áreas $\mathrm{A}_{2}$ e $\mathrm{A}_{3}$ nas profundidades de $10-20 \mathrm{~cm}$ e de $20-30 \mathrm{~cm}$ (Tabela 2). No total, foram apenas nove emergências no escuro, e todas da mesma espécie, a Brachiaria sp. Em geral, sementes pequenas com poucas reservas, bem como de espécies arbóreas pioneiras pertencentes à parte superior de uma floresta apresentam fotodormência (fotoblásticas positivas). Essa dormência sob o dossel proporciona a formação de bancos de sementes no interior da floresta, permitindo a regeneração natural das espécies, quando ocorrem aberturas de clareiras. Isso demonstra a importância do banco de sementes e das suas espécies constituintes.

TABELA 2: Número médio de sementes germinadas nas áreas $A_{1}, A_{2}$ e $A_{3}$, correspondentes a área degradada do Parque do Mocambo em diferentes profundidades de solo. $\mathrm{S}_{1}$ correspondente ao solo coletado entre $0-10 \mathrm{~cm}, \mathrm{~S}_{2}$ entre $10-20 \mathrm{~cm}$ e $\mathrm{S}_{3}$ de $20-30 \mathrm{~cm}$.

\begin{tabular}{cccc}
\hline \multicolumn{4}{c}{ Áreas submetidas ao escuro } \\
\hline Profundidade & $\mathbf{A}_{1}$ & $\mathbf{A}_{2}$ & $\mathbf{A}_{3}$ \\
\hline $\mathbf{S}_{1}$ & $0,00 \mathrm{a}^{*}$ & $0,33 \mathrm{a}$ & $0,22 \mathrm{a}$ \\
$\mathbf{S}_{2}$ & $0,00 \mathrm{a}$ & $0,11 \mathrm{a}$ & $0,22 \mathrm{a}$ \\
$\mathbf{S}_{3}$ & $0,00 \mathrm{a}$ & $0,00 \mathrm{a}$ & $0,11 \mathrm{a}$ \\
\hline
\end{tabular}

* médias seguidas pelas mesmas letras não diferem entre si pelo teste Kruskal-Wallis.

Nas aberturas de clareiras, as sementes fotoblásticas positivas germinam após receberem um pulso de luz vermelha (V). Este evento é iniciado pelos processos envolvendo o fitocromo e o ácido giberélico. A ação da luz consiste basicamente em transformar a forma inativa do fitocromo (Fv) para a forma ativa (Fve). A luz solar faz com que os valores de Fve aumentem até um limite em que a dormência seja quebrada. O fitocromo ativo (Fve) é responsável pela expressão gênica que conduz à síntese do ácido giberélico (GA), que é promotor da germinação. Já o fitocromo inativo (Fv) é responsável pela síntese do ácido abscísico ou abscisina (ABA), um inibidor da germinação (TAIZ; ZEIGER, 2004).

Relacionando as Tabelas 1 e 2 deste trabalho, nota-se ainda que a porção mais superficial do solo $\mathrm{S}_{1}$ $(0-10 \mathrm{~cm})$, foi onde ocorreu a maior taxa de germinação, tanto para as amostras no claro quanto para as no escuro, e a área $\mathrm{A}_{3}$ obteve o maior índice de germinações. A ausência de germinações nas camadas mais profundas pode significar que não houve depósito de sementes em longo prazo, germinando somente as mais superficiais, ou que as sementes mais profundas tenham perdido a sua viabilidade. Nesse caso, o revolvimento do solo não seria uma prática relevante para melhorar a germinação no banco de sementes.

De acordo com as análises das amostras do solo, as diferentes áreas e profundidade revelaram que o pH em água varia entre 6,47 - 7,10 (Tabela 3). Isto indica que, solos com $\mathrm{pH}$ acima de 6 , não é necessário a aplicação de corretivos. A acidez do solo refere-se à sua capacidade de liberar prótons, variando o gradiente de concentração no ambiente (VELOSO et al., 1992). De acordo com Ribeiro et al. (1999), os níveis de $\mathrm{Ca}^{2+}$ e $\mathrm{Mg}^{2+}$ encontram-se bons em $\mathrm{A}_{1}$ e $\mathrm{A}_{2}$, e muito bons em $\mathrm{A}_{3}$. Os demais componentes, como o CTC (capacidade de troca de cátions), SB (soma de bases), m (saturação por $\mathrm{Al}^{3+}$ ) e V (saturação por bases) demonstraram teores que variam entre bons e muito bons, com exceção do $\mathrm{P}$ e $\mathrm{K}^{+}$, que apresentaram valores baixos (Tabela 3 ).

A capacidade de reflorestamento do local de estudo está diretamente ligada ao seu banco de sementes e a fertilidade do solo. A velocidade de regeneração das florestas e matas depende da intensidade da perturbação sofrida (TABARELLI; MONTAVANI, 1999). Através dos dados obtidos nesse trabalho, foi possível afirmar que estas áreas do Parque do Mocambo em estudo possuem capacidade de regeneração natural. De acordo com o levantamento florístico, há presença de espécies arbóreas 
TABELA 3: Valores médios de M.O. (matéria orgânica), K, Al, Mg, SB (soma de bases), Ca, t (CTC efetiva), T (CTC pH 7), V (saturação por bases), $\mathrm{m}$ (saturação por $\mathrm{Al}^{3+}$ ) de amostras do solo relacionando as três áreas estudadas e as três profundidades em cada área.

\begin{tabular}{|c|c|c|c|c|c|c|c|c|c|c|c|c|}
\hline \multirow{2}{*}{$\begin{array}{c}\text { Área/ } \\
\text { profund. }\end{array}$} & pH & P-Meh & $\mathbf{K}$ & $\mathrm{Ca}^{2+}$ & $\mathbf{M g}^{2+}$ & $\mathbf{A \mathbf { l } ^ { 3 + }}$ & $\mathbf{H}+\mathbf{A l}$ & SB & t & $\mathbf{T}$ & \multirow{2}{*}{$\frac{\mathrm{V}}{\%}$} & \multirow{2}{*}{$\frac{\text { M.O }}{\text { dag kg- }}$} \\
\hline & Água & \multicolumn{2}{|c|}{$\mathrm{mg} \mathrm{dm}{ }^{-3}$} & \multicolumn{7}{|c|}{$\mathrm{cmol}_{\mathrm{c}} \mathrm{dm}^{-3}$} & & \\
\hline $\mathrm{A}_{1} \mathrm{~S}_{1}{ }^{1}$ & 6,47 & 0,88 & 13,49 & 4,0 & 2,3 & 0,1 & 2,6 & 6,33 & 6,43 & 8,93 & 71 & 4,64 \\
\hline $\mathrm{A}_{1} \mathrm{~S}_{2}$ & 6,75 & 0,88 & 10,65 & 3,8 & 3,0 & 0,1 & 2,6 & 6,83 & 6,93 & 9,43 & 72 & 3,51 \\
\hline $\mathrm{A}_{1} \mathrm{~S}_{3}$ & 6,88 & 0,43 & 24,34 & 3,9 & 4,4 & 0,2 & 2,4 & 8,36 & 8,56 & 10,76 & 78 & 3,77 \\
\hline $\mathrm{A}_{2 \mathrm{~S} 1}$ & 6,53 & 1,15 & 16,84 & 4,4 & 3,8 & 0,0 & 2,3 & 8,24 & 8,24 & 10,54 & 78 & 5,90 \\
\hline $\mathrm{A}_{2} \mathrm{~S}_{2}$ & 6,48 & 0,85 & 15,31 & 3,0 & 3,6 & 0,1 & 3,3 & 6,64 & 6,74 & 9,94 & 67 & 4,52 \\
\hline $\mathrm{A}_{2} \mathrm{~S}_{3}$ & 6,72 & 1,61 & 9,63 & 3,8 & 2,9 & 0,2 & 3,0 & 6,72 & 6,92 & 9,72 & 69 & 4,77 \\
\hline $\mathrm{A}_{3} \mathrm{~S}_{1}$ & 7,10 & 1,71 & 45,0 & 6,0 & 1,9 & 0,2 & 3,2 & 8,02 & 8,22 & 11,22 & 71 & 5,52 \\
\hline $\mathrm{A}_{3} \mathrm{~S}_{2}$ & 6,97 & 1,23 & 19,27 & 5,5 & 1,0 & 0,1 & 2,2 & 6,55 & 6,65 & 8,75 & 75 & 4,27 \\
\hline $\mathrm{A}_{3} \mathrm{~S}_{3}$ & 7,02 & 1,73 & 53,75 & 5,1 & 3,6 & 0,1 & 3,0 & 8,84 & 8,84 & 11,84 & 75 & 4,90 \\
\hline
\end{tabular}

${ }^{1}$ Áreas: $A_{1}$ (topo), $A_{2}$ (encosta) e $A_{3}$ (baixada); Profundidade do solo: $\mathrm{S}_{1}($ de $0-10 \mathrm{~cm}) ; \mathrm{S}_{2}(\mathrm{de} 10-20 \mathrm{~cm}) ; \mathrm{S}_{3}(\mathrm{de} 20-30 \mathrm{~cm})$.

no local, porém no banco de sementes analisado foram encontradas apenas espécies pioneiras herbáceas. Esse fato pode ser justificado em virtude de que as plantas arbóreas presentes na área de estudo ainda não entraram em fase reprodutiva ou estas espécies apresentam algum tipo dormência, ou pode ser também pela ausência de dispersores naturais das mesmas. Provavelmente, essas áreas consigam se reintegrar ecologicamente em longo prazo, e principalmente quando auxiliada com a correção de potássio e fósforo, bom como, da introdução de espécies lenhosas do cerrado, tal como Casearia sylvestris, Dalbergia miscolobium, Kielmeyera coreacea, Ouratea hexasperma e Salacia crassifolia, que apresentam alta capacidade de recuperação de áreas perturbadas (SENA; PINTO, 2008).

A partir dos resultados, foi possível concluir que: 1) Houve germinação de espécies pioneiras herbáceas, e em sua maioria fotoblásticas positivas, tendo maior representatividade na profundidade $\left.0-10 \mathrm{~cm}\left(\mathrm{~S}_{1}\right) ; 2\right) \mathrm{A}$ análise do solo mostrou valores satisfatórios em quase todos os componentes, com exceção do potássio e do fósforo. De acordo com a taxa de germinação encontrada, pode-se afirmar que a capacidade de regeneração natural existe, porém sugere-se a realização de trabalhos futuros para acelerar a recuperação do Parque do Mocambo em consórcio com plantio de espécies arbóreas e também atrativos para dispersores de sementes.

\section{Referências}

AGUiAR, L. M. S.; CAMARGO, A. J. A. Cerrado: ecologia e caracterização. Brasília: Embrapa Informações Tecnológica, 2004. 249 p.

ALMEIDA-CORTEZ, J. S. Dispersão e banco de sementes. In: FERREIRA, A. G.; BORGUETTI, F. (Orgs). Germinação: do básico ao aplicado. Porto Alegre: Artmed, 2004. p. 225-235.

ARAÚJO, M. M.; LONGHI, S. J.; BARROS, P. L. C.; BRENA, D. A. Caracterização da chuva de sementes, banco de sementes do solo e banco de plântulas em Floresta Estacional decidual ripária Cachoeira do Sul, RS. Scientia Forestalis, Piracicaba, v. 66, p.128141, 2004.

BAIDER, C.; MANTOVANI, W.; TABARELLI, M. O banco de sementes de um trecho da floresta Atlântica Montana. Revista Brasileira de Biologia, São Carlos, v. 59, p. 319-328, 1999.

BRECHARA, F. C.; CAMPOS-FILHO, E. M. C.; BARRETTO, K. D.; GABRIEL, V. S.; ANTUNES, A. Z.; REIS, A. Unidades demonstrativas de restauração ecológica através de técnicas nucleadoras de biodiversidade. Revista Brasileira de Biociências, Porto Alegre, v. 5, n. 1, p. 9-11, 2007.

CALDATO, S. L.; FLOSS, P. A.; CROCE, D. M.; LONGHI, S. J. Estudo da regeneração natural, banco de sementes e chuva de sementes na reserva genética florestal de Caçador, SC. Ciência Florestal, Santa Maria, v. 6, n. 1, p. 27-38, 1996.

CHRISTOFFOLETI, P. J.; CAETANO, R. S. X. Soil seed banks. Scientia Agricola, Piracicaba, v. 55, p. 74-78, 2008.

GASPARINO, D.; MALAVASI, U. C.; MALAVASI, M. M.; SOUZA, I. Quantificação do banco de sementes sob diferentes usos do solo em área de domínio ciliar. Revista Árvore, Viçosa, v. 30, n. 1, p. 1-9, 2006.

LORENZI, H. Plantas daninhas do Brasil: terrestres, aquáticas, parasitas e tóxicas. 2 ed. Nova Odessa: Instituto Plantarum, 2000. $624 \mathrm{p}$. 
NÓBREGA, A. M. F.; VALERI, S. V.; PAULA, R. C.; PAVANI, M. C. M. D.; SILVA, S. A. Banco de sementes de remanescentes naturais e de áreas reflorestadas em uma várzea do Rio Mogi-Guaçu - SP. Revista Árvore, Viçosa, v. 33, n. 3, p. 403-411, 2009.

MIRANDA, M. D.; OLIVIERA, U. ; GARCIA, Q. Z. Avaliação do banco de sementes em dois remanescentes de mata atlântica no Parque Estadual do Rio Doce - MG. In: CONGRESSO DE ECOLOGIA DO BRASIL, 8, 2007, Caxambu. Anais... Caxambu: CEB, 2007. Versão eletrônica.

ODUM, E. P. Fundamentos de Ecologia. 4 ed. Lisboa: Fundação Calouste Gulbenkian, 1988. 459 p.

PATOS DE MINAS. Lei n. 2.598 de 28 de março de 1990. Declara de preservação permanente área de proteção a nascente do córrego da Caixa D'agua e do Parque do Mocambo. Lex: Câmara Municipal de Patos de Minas, p.1-4, mai., 1trim 1990.

PINTO JÚNIOR, R. A. Potencial da chuva de sementes, da regeneração natural e da transposição do solo na recuperação de pastagem degradada. 2008. 42 f. Monografia (Graduação em Gestão Ambiental) - Escola Agrotécnica Federal de Inconfidentes, Inconfidentes. 2008.

REZENDE, A. V. Importância das matas de galeria: manutenção e recuperação. In: RIBEIRO, J. F. (Ed.). Cerrado: matas de galeria. Planaltina: EMBRAPA-CPAC, 1998. p. 3-16.

RIBEIRO, A. C.; GUIMARÃES, P. T. G.; AlvAREZ, V. H. Recomendações para uso de corretivo e fertilizantes em Minas Gerais. Viçosa: Comissão de Fertilidade do Solo do Estado de Minas Gerais, 1999. 359 p.

SENA, A. L. M.; PINTO, J. R. R. Regeneração natural em áreas degradadas com enfoque na capacidade de resiliência das espécies lenhosas do cerrado. In: SIMPÓSIO NACIONAL DO CERRADO, 10, 2008, Brasília. Resumos... Brasília: SNC, 2008. Disponível em $<$ simposio.cpac.embrapa.br/simposio_pc210/fichas/00278_trab1 ficha.pdf $>$.
SILVA, F. A. S. E. The Assistat software: statistical assistance. In: INTERNATIONAL CONFERENCE ON COMPUTERS IN AGRICULTURE, Cancun, 1996. Anais... Cancun: American Society of Agricultural Engineers, 1996. Versão eletrônica.

TABARELLI, M.; MANTOVANI, W. A regeneração de uma floresta tropical montana após corte e queima (São Paulo - Brasil). Revista Brasileira de Biologia, São Paulo, v. 59, n. 2, p. 239-250, 1999.

TAIZ, L.; ZIEGER, E. Fisiologia Vegetal. 3 ed. Porto Alegre: Artemed, 2004. 719 p.

TOMAZI, A. L.; ZIMMERMANN, C. E.; LAPS, R. R. Poleiros artificiais como modelo de nucleação para restauração de ambientes ciliares: caracterização da chuva de sementes e regeneração natural. Biotemas, Florianópolis, v. 23, n. 3, p. 125-135, 2010.

VALLEJO, L. R. Unidades de Conservação: uma discussão teórica à luz dos conceitos de território e de políticas públicas. GEOgraphia, Rio de Janeiro, v. 4, n. 8, p. 77-106, 2003.

VELOSO, C. A. C.; BORGES, A. L.; MUNIZ, A. S.; VEIGAS, I. A. DE J. M. Efeito de diferentes materiais no $\mathrm{pH}$ do solo. Scientia Agricola, Piracicaba, v. 49, n. 1, p. 123-128, 1992.

VIEIRA, N. K.; REIS, A. O papel do banco de sementes na restauração de áreas degradadas. In: SEMINÁRIO NACIONAL, 2003, Foz do Iguaçu, Anais... Foz do Iguaçu: ASN, 2003.

VINHA, D. Banco de sementes em áreas com diferentes graus de perturbação no Parque Estadual das Fontes do Ipiranga, em São Paulo, SP. 2008. 105 f. Tese (Mestrado em Botânica) Instituto de Botânica de São Paulo, São Paulo. 2008. 